\title{
Leiomiossarcoma primário da glândula tireoide
}

\author{
Thyroid gland primary leiomyosarcoma
}

Antonio Augusto T. Bertelli', Luiz Cláudio Bosco Massarollo², Erivelto Martinho Volpi ${ }^{3}$, Rubens Yassuzo Ykko Ueda ${ }^{4}$, Elci Barreto ${ }^{5}$

\footnotetext{
' Disciplina de Cirurgia de Cabeça e Pescoço, Departamento de Cirurgia, Faculdade de Ciências Médicas da Santa Casa de São Paulo (FCMSC-SP), São Paulo, SP, Brasil ${ }^{2}$ Serviços de Cirurgia de Cabeça e Pescoço, Hospital Carlos Chagas e Instituto de Oncologia de Guarulhos, Garulhos, SP, Brasil ${ }^{3}$ Serviço de Cirurgia de Cabeça e Pescoço, Hospital das Clínicas, Faculdade de Medicina, Universidade de São Paulo (FMUSP), São Paulo, SP, Brasil ${ }^{4}$ Serviço de Cirurgia de Cabeça e Pescoço, Hospital Carlos Chagas, Garulhos, SP, Brasil ${ }^{5}$ Departamento de Patologia, Faculdade de Medicina do ABC (FMABC), Santo André, SP, Brasil
}

Correspondência para: Antonio Augusto T. Bertelli Rua Treze de Maio, $1.856,4^{\circ}$ andar 01327-002 - São Paulo, SP antonio.bertelli@terra.com.br

Recebido em 11/Maio/2009 Aceito em 2/Nov/2009

\section{SUMÁRIO}

Aproximadamente $15 \%$ a $20 \%$ dos sarcomas ocorrem na região da cabeça e pescoço, $80 \%$ em adultos, sendo apenas $0,014 \%$ leiomiossarcomas primários de tireoide. Existem apenas 16 casos relatados no mundo, dos quais não há nenhum em nosso meio. São tumores com diagnóstico citológico pré-operatório difícil e podem ser confundidos com outras lesões mais comuns da tireoide, como carcinomas anaplásicos e medulares. 0 tratamento ideal ainda não está bem definido, visto que o prognóstico é ruim e a cirurgia radical associada à quimioterapia e à radioterapia adjuvantes não demonstra melhora nas taxas de recorrência e sobrevida. Relatou-se um caso de leiomiossarcoma primário da glândula tireoide em um paciente jovem, submetido a tireoidectomia total e esvaziamento cervical, associado à radioterapia adjuvante e realizou-se uma extensa revisão da literatura existente sobre o tema. Houve boa evolução pós-operatória, sem sinais de recidiva após quatro anos de seguimento. Arq Bras Endocrinol Metab. 2010;54(3):326-30

\section{SUMMARY}

Despite the fact that $15 \%$ to $20 \%$ of sarcomas occur in the head and neck and $80 \%$ in adults, only $0.014 \%$ are primary thyroid leiomyosarcomas. To the best of our knowledge, only 16 cases have been reported around the world, none in South America. Cytologic diagnosis is challenging and these tumors may be mistaken by more common ones such as anaplastic or medullary carcinomas. The treatment of choice for thyroid leiomyosarcomas is not well established yet because of its poor prognosis. Radical surgery associated with chemoradiotherapy has not been effective and did not improve survival rates. The authors report a case of primary thyroid leiomyosarcoma in a young male, who has been submitted to total thyroidectomy and selective neck dissection. Extensive literature review was performed by the authors. The patient received adjuvant radiotherapy, presenting good postoperative course. After four years evolution, there was no local recurrence or distant metastasis. Ara Bras Endocrinol Metab. 2010;54(3):326-30
$\mathrm{O}$ sarcomas correspondem a menos de $1 \%$ dos tumores malignos que acometem a cabeça e o pescoço em adultos (1). O leiomiossarcoma responde por apenas $2,3 \%$ a $5,3 \%$ dos tumores malignos dos tecidos moles, acometendo preferencialmente o trato genital feminino (sobretudo o útero), trato gastrointestinal, retroperitônio e tecido subcutâneo (2). Aproximadamente $1 \%$ dos sarcomas de cabeça e pescoço é leiomiossarcoma, ocorrendo principalmente na cavidade oral, nos tecidos moles superficiais, como couro cabeludo, seios paranasais e mandíbula (3); o acometimento da tireoide é extremamente raro (4). Existem apenas 16 casos relatados no mundo, dos quais não há nenhum deles em nosso meio. São tumores com diagnóstico citológico pré-operatório difícil e podem ser confundidos com outras lesões mais comuns da tireoide, como carcinomas anaplásicos e medulares (5). Alguns autores acreditam que os sarcomas tireoideanos são carcinomas anaplásicos com diferenciação sarcomatosa (6), no entanto estudos de imuno-histoquímica e microscopia eletrônica confirmam a existência de leiomiossarcomas primários de tireoide, que se originam possivelmente a partir da musculatura lisa da parede vascular (7-11). $\mathrm{O}$ tratamento ideal ainda não está bem definido, visto 
que o prognóstico desses tumores é ruim e a cirurgia radical associada à quimioterapia e à radioterapia adjuvantes não demonstrara melhora nas taxas de recorrência e sobrevida (5-14). Relatou-se um caso de leiomiossarcoma primário da glândula tireoide em um paciente do gênero masculino, jovem, com nódulo tireoideano de crescimento rápido, submetido à tireoidectomia total e ao esvaziamento cervical, associado à radioterapia adjuvante. Apresentou boa evolução pós-operatória, sem sinais de recidiva após quatro anos de seguimento. Realizou-se ainda uma extensa revisão da literatura existente sobre o tema.

\section{RELATO DE CASO}

Relatamos o caso de um paciente com 39 anos de idade, gênero masculino, com queixa de nódulo cervical anterior com crescimento progressivo havia 4 meses, acompanhado de disfonia e desconforto cervical, sem antecedente de nenhuma outra neoplasia ou de irradiação cervical prévia. O paciente procurou inicialmente um otorrinolaringologista que, por meio de laringoscopia, evidenciou pregas vocais móveis e encaminhou-o aos nossos cuidados. Ao exame, apresentava nódulo de
$3,5 \mathrm{~cm}$ na topografia do lobo direito da tireoide, móvel à deglutição e de consistência fibroelástica. Não havia linfonodomegalias cervicais palpáveis e o restante do exame clínico não demonstrava alterações. A ultrassonografia confirmou o achado do nódulo tireoideano, sem evidências de nódulos contralaterais. A punção aspirativa por agulha fina demonstrou suspeita de malignidade, mas sem sugerir o tipo histológico. A calcitonina sérica era normal, assim como a função tireoideana. Foi optado pela realização de tireoidectomia total, com esvaziamento cervical seletivo do nível VI ipsilateral à lesão, por cervicotomia a Kocher. No intraoperatório, observou-se lesão nodular de $3,5 \mathrm{~cm}$ localizada internamente à cápsula, sem extravasamento macroscópico, substituindo todo o lobo direito da tireoide e com plano de clivagem entre as estruturas adjacentes. $\mathrm{O}$ exame perioperatório de congelação revelou neoplasia maligna fusocelular. $\mathrm{O}$ estudo anatomopatológico evidenciou neoplasia maligna, pouco diferenciada, caracterizada por células anaplásicas alongadas exibindo acentuado pleomorfismo nuclear e hipercromasia (Figura 1). De permeio apresentava focos de necrose e índice mitótico superior a 5/CGA. A neoplasia, não encapsulada, estava circundada por tecido fibroadiposo, não comprometendo

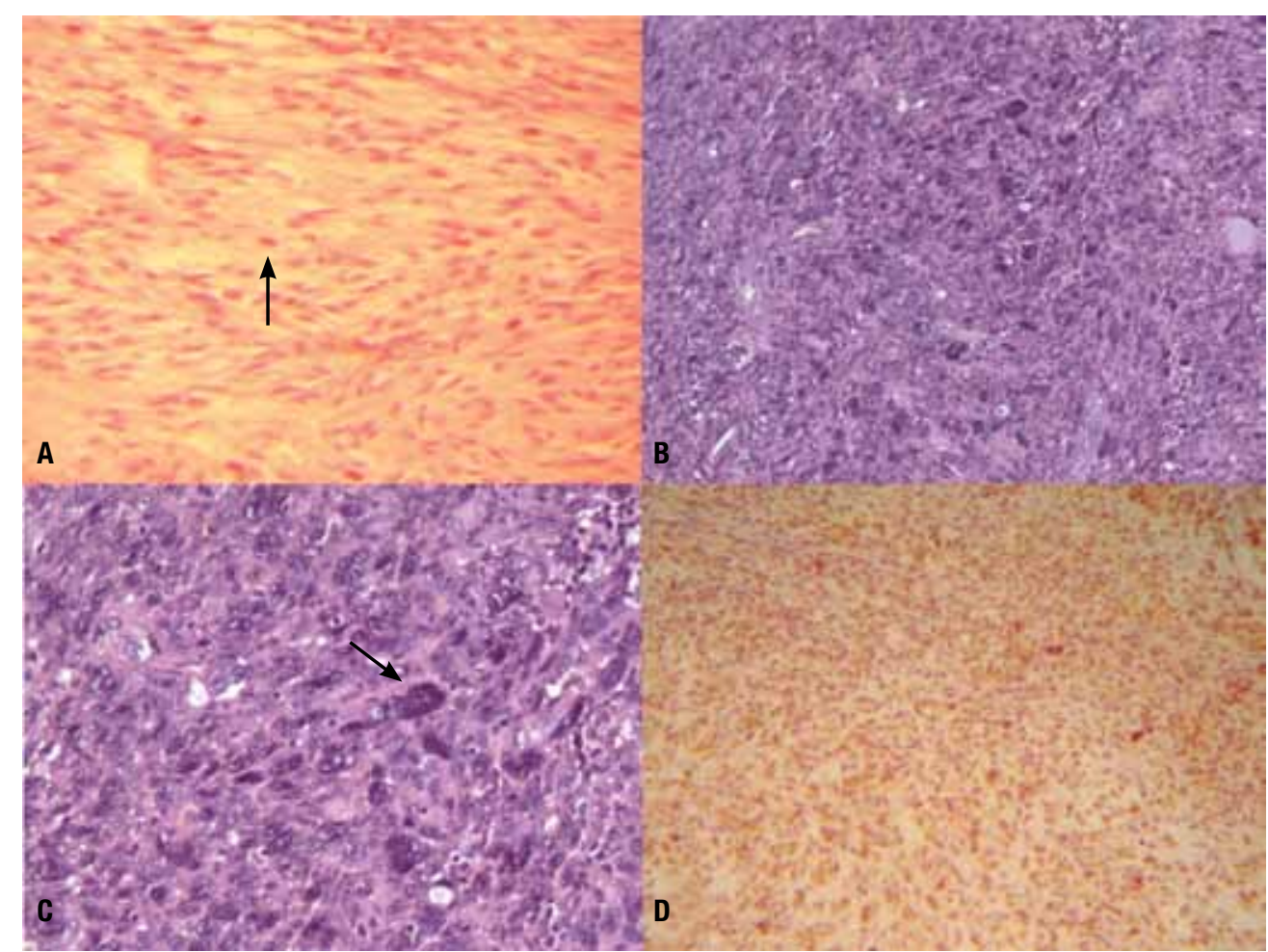

Figura 1. Fotomicrografias de leiomiossarcoma primário da glândula tireoide. A: neoplasia fusocelular com núcleos hipercromáticos (seta) (hematoxilinaeosina - 100X). B: as células tumorais exibem intensa anaplasia (hematoxilina-eosina - 100X). C: os núcleos apresentam pleomorfismo e hipercromasia (seta) (hematoxilina-eosina - 400X). D: células tumorais com imuno-histoquímica positiva para actina musculoespecífica (HHF-35 - 40X). 
as margens cirúrgicas. Não se detectou infiltração perineural ou invasão vascular. $\mathrm{O}$ parênquima tireoideano circunjacente exibia folículos preservados. A avaliação imuno-histoquímica pelo método da estreptavidinabiotina-peroxidase com utilização de anticorpos mono e policlonais incluiu pesquisa de citoqueratina de alto peso molecular, citoqueratina $\mathrm{AEl} / \mathrm{E} 3$, proteína $\mathrm{S} 100$ e CD 68 que resultaram negativos. A pesquisa de actina musculoespecífica (HHF-35) e desmina foi positiva (Figura 1), o que caracterizou perfil imuno-histológico consistente com leiomiossarcoma grau II. Não havia metástases linfonodais no produto do esvaziamento cervical. O estadiamento por imagem com tomografia de tórax e crânio, ultrassonografia de abdome e mielograma foi negativo. $\mathrm{O}$ paciente foi submetido à radioterapia externa adjuvante com dose de 7.000 CGy em campos cervicais e não recebeu quimioterapia. Encontra-se aos 48 meses de seguimento, sem sinais de recidiva local ou de metástases a distância.

\section{DISCUSSÃO}

Aproximadamente $15 \%$ a $20 \%$ dos sarcomas ocorrem na região da cabeça e pescoço, $80 \%$ em adultos, sendo apenas $0,014 \%$ leiomiossarcomas primários de tireoide
$(6,13)$. O primeiro caso descrito em 1969 por Adachi e cols. (15) foi um leiomiossarcoma primário de tireoide com metástases para o coração e cérebro. Desde então, foram descritos apenas 17 casos, incluindo o atual, sendo seis do gênero masculino e 10 , do feminino ( 1 caso sem informação sobre o sexo), com idades variando entre 39 e 90 anos e mediana de 66 anos (4-16) (Tabela 1).

Ramakrishnan e cols. (17) descreveram um caso de leiomiossarcoma primário da tireoide em uma criança de 3 anos. Entretanto, durante a cirurgia não parecia haver invasão da glândula tireoide e concordou-se com Just e cols. (4) em não considerar tal tumor como primário da tireoide, mas sim da região cervical. Tulbah e cols. (18) descreveram um caso de leiomiossarcoma multifocal da tireoide, fígado e pulmão, associado com o vírus Epstein Barr (EBV) em um menino de 6 anos com imunodeficiência congênita de células T. Embora alguns autores tenham demonstrado a associação do EBV com tumores de células musculares lisas em crianças com HIV e transplantadas imunossuprimidas (18), o fato de a neoplasia descrita ser multifocal não afasta a possibilidade de uma metástase de um leiomiossarcoma primário do fígado ou do pulmão para a glândula tireoide. Portanto, também não se considerou esse caso como um tumor primário da glândula tireoide.

Tabela 1. Dados clínicos referentes aos 17 casos de leiomiossarcoma primário da glândula tireoide

\begin{tabular}{|c|c|c|c|c|c|c|c|c|c|c|}
\hline Autor & Ano & Sexo & Idade & $\mathrm{T}(\mathbf{c m})$ & cirurgia & Tratamento (outro) & SV & rec & M1 & Status \\
\hline Bertelli* & 2009 & M & 39 & 3,5 & $\mathrm{TT}+\mathrm{EC}$ & Radioterapia & $48 m$ & $\mathrm{~N}$ & $\mathrm{~N}$ & VSD \\
\hline Wang e cols. (5) & 2008 & $\mathrm{~F}$ & 65 & 7,5 & $\mathrm{TT}+\mathrm{EC}$ & Ifosfamida/adriamicina & $4 \mathrm{~m}$ & $\mathrm{~N}$ & $\mathrm{~N}$ & VSD \\
\hline Mansouri e cols. (14) & 2008 & $\mathrm{~F}$ & 63 & 7 & TT & $x x$ & $5 \mathrm{~m}$ & $\mathrm{~N}$ & S & MPD \\
\hline Just e cols. (4) & 2008 & $\mathrm{~F}$ & 83 & 9 & $x x$ & Cuidados paliativos & $2 m$ & $x x$ & $\mathrm{~N}$ & MPD \\
\hline Day e cols. (12) & 2007 & M & 43 & 6 & $\mathrm{TT}+\mathrm{EC}$ & Imatinibe & $6 \mathrm{~m}$ & $S$ & $S$ & MPD \\
\hline Takayama e cols. (13) & 2001 & $F$ & 66 & 8,5 & $\mathrm{TsT}+\mathrm{LT}$ & $x x$ & $3 m$ & S & $S$ & VCD \\
\hline Tsugawa e cols. (10) & 1999 & $\mathrm{~F}$ & 90 & 8 & Tumorectomia + TQT & $x x$ & $2 m$ & $x x$ & $x x$ & MPD \\
\hline \multirow[t]{4}{*}{ Thompson e cols. (6) } & 1997 & $\mathrm{~F}$ & 64 & 7,5 & Tumorectomia & $x x$ & $5 \mathrm{~m}$ & $x x$ & $S$ & MPD \\
\hline & & M & 45 & 9 & Hemitireoidectomia & Doxorrubicina & $11 \mathrm{~m}$ & $\mathrm{~N}$ & $S$ & VCD \\
\hline & & M & 68 & 1,9 & Tumorectomia & $x x$ & $18 m$ & $x x$ & S & MPD \\
\hline & & M & 83 & 5,5 & TT & $x x$ & $3 \mathrm{~m}$ & $\mathrm{~N}$ & S & MPD \\
\hline Ozaki e cols. (9) & 1997 & $F$ & 58 & 5 & $\mathrm{TT}+\mathrm{EC}$ & $x x$ & $25 \mathrm{~m}$ & $\mathrm{~N}$ & $\mathrm{~N}$ & VSD \\
\hline Chetty e cols. (11) & 1993 & $\mathrm{~F}$ & 54 & 3,5 & Lobectomia & $x x$ & $15 \mathrm{~m}$ & $\mathrm{~N}$ & $\mathrm{~N}$ & VSD \\
\hline lida e cols. (8) & 1993 & $\mathrm{~F}$ & 72 & 3 & Lobectomia + EC & $x x$ & $51 \mathrm{~m}$ & N & S & MPD \\
\hline Kaur e Jayaram (16) & 1990 & $x x$ & $x x$ & $x x$ & $x x$ & $x x$ & $12 m$ & $x x$ & $x x$ & VCD \\
\hline Kawahara e cols. (7) & 1988 & M & 82 & 5,5 & Lobectomia + EC & $x x$ & $4 \mathrm{~m}$ & $S$ & $\mathrm{~N}$ & MPD \\
\hline Adachi e cols. (15) & 1969 & $F$ & 74 & 12 & $x x$ & Quimioterapia & $1 \mathrm{~m}$ & $x x$ & S & MPD \\
\hline
\end{tabular}

* Caso atual; EC: esvaziamento cervical; LT: laringectomia total; M1: metástase a distância; MPD: morto pela doença; rec: recidiva local; SV: sobrevida; T: tamanho do tumor; TQT: traqueostomia; TST: tireoidectomia subtotal; TT: tireoidectomia total; VCD: vivo com doença; VSD: vivo sem doença; XX: ausente ou não informado. 
O diagnóstico de leiomiossarcoma primário da tireoide requer muita prudência. Além de leiomiossarcomas da região cervical serem mais comuns que os primários da tireoide e poderem invadir a glândula, $o$ tumor primário da tireoide também pode invadir estruturas adjacentes extravasando a cápsula tireoideana, causando uma confusão diagnóstica. Ademais, deve ser excluída a hipótese de metástase. Isso só é possível por meio de exames clínico e de imagem cuidadosos para afastar a possibilidade de um leiomiossarcoma primário oculto em outro órgão (6).

A queixa principal geralmente é de aumento rápido do volume cervical, algumas vezes associado a disfagia, dispneia, disfonia e perda de peso (4), que pode levar ao diagnóstico presumido de carcinoma indiferenciado da tireoide ou de linfoma primário da tireoide, principalmente nos indivíduos idosos (13).

A ultrassonografia da tireoide geralmente é inespecífica e confirma o achado do exame clínico (5). A tomografia computadorizada do pescoço pode demonstrar uma tumoração hipodensa com necrose e calcificações, além de poder evidenciar invasão de estruturas vizinhas como a cartilagem tireoide, veia jugular interna, artéria carótida, musculatura pré-tireoideana e esôfago $(4,5,9,13)$. Just e cols. (4) descreveram um caso de leiomiossarcoma tireoideano com invasão da primeira vértebra torácica e sintomas neurológicos no membro superior. A ressonância magnética pode demonstrar uma tumoração de sinal intermediário em T2 e isointenso em Tl, com realce claro ao gadolínio (13).

A punção aspirativa por agulha fina pré-operatória geralmente não revela o tipo do tumor, quando se trata de um leiomiossarcoma, mas ela pode demonstrar células fusiformes com anaplasia e sugerir o diagnóstico de neoplasia maligna (12), como ocorreu no caso relatado. Entre os principais diagnósticos diferenciais, encontramse carcinoma medular, carcinoma indiferenciado, sarcoma sinovial, tumor fibroso solitário e fibrossarcoma (5).

Os leiomiossarcomas tireoideanos tendem a ser grandes, com necrose e hemorragia à macroscopia. À microscopia, têm padrão de crescimento fascicular e multidirecional, grau de atipias muito variável e podem ter células gigantes tumorais de permeio, além de alterações mixoides (4). A maioria dos autores concorda que os leiomiossarcomas tireoideanos originam-se na camada muscular de vasos sanguíneos, assim como foi proposto para outros tumores de músculo liso que ocorrem em outras localizações não usuais (6). Adachi e cols. (15) constataram que o primeiro caso de leiomiossarco- ma primário da tireoide, descrito em uma necropsia, se originava nas paredes vasculares da cápsula tireoideana, visto que não existe musculatura lisa no ducto tireoglosso. Kawahara e cols. (7) descreveram, pela primeira vez, as alterações imuno-histoquímicas de um leiomiossarcoma primário da tireoide e corroboraram tal proposição. O perfil imuno-histoquímico inclui positividade para vimentina, actina musculoespecífica, miosina, desmina, e negatividade para citoqueratina, tireoglobulina, calcitonina, proteína S100 e cromogranina, e é decisivo para a confirmação diagnóstica (4-6,11-13,19).

Muitos estudos, com o auxílio da imuno-histoquímica e microscopia eletrônica, demonstraram que a maioria dos tumores sarcomatosos da glândula tireoide são carcinomas indiferenciados com diferenciação sarcomatosa, ou seja, de origem epitelial e não mesenquimal. Tais tumores podem demonstrar expressão de vimentina e citoqueratina, além de expressão ocasional de tireoglobulina, mas não demonstram expressão de desmina, actina musculoespecífica, cromogranina ou calcitonina (6). A classificação de tumores de tireoide da Organização Mundial da Saúde indica o leiomioma e o leiomiossarcoma como as duas variantes possíveis para os tumores de músculo liso primários da tireoide (19).

Seu tratamento principal é cirúrgico em decorrência da baixa eficácia da quimioterapia e da radioterapia nestes casos, além da tendência do tumor à infiltração de estruturas adjacentes, o que torna o tratamento oncológico radical essencial para o controle local do tumor $(4,5,12)$. Nos casos relatados na literatura, foram realizadas cirurgias em 14 dos 17 pacientes, entre elas: tireoidectomia parcial ou subtotal em cinco, tireoidectomia total em seis e tumorectomia em três casos. O tamanho do tumor variou entre 1,9 e $12,0 \mathrm{~cm}$ com média de $6,4 \mathrm{~cm}$ e geralmente substitui todo o lobo tireoideano (4), como no caso relatado. O esvaziamento cervical foi associado a um desses procedimentos em seis pacientes, a traqueostomia em um e a laringectomia total em outro. O paciente do presente caso foi submetido à tireoidectomia total com esvaziamento cervical seletivo do nível VI homolateral à lesão. As metástases cervicais são menos frequentes que nos carcinomas medulares e indiferenciados (7-13,15): apenas três casos relatados na literatura apresentaram metástases linfonodais $(7,15,16)$. O caso exposto não apresentou metástases linfonodais no momento da cirurgia ou mesmo no seguimento realizado. A quimioterapia foi utilizada isoladamente em um caso e de maneira adjuvante em três. Em nenhum caso a radioterapia externa foi utilizada 
como tratamento isolado ou adjuvante $(4-13,15,16)$, como no caso em questão. Todos os casos estão pormenorizados na tabela 1 .

Entre os casos descritos até os dias de hoje, a sobrevida variou entre 1 e 51 meses. A despeito do tratamento empregado, a frequência de metástases a distância chega a mais de $50 \%$ (9 dos 16 pacientes) (6,8,12-15), tendo entre os principais sítios os pulmões e o fígado, além de rim, pâncreas, intestino delgado, peritônio, cólon, miocárdio, ossos e cérebro $(4,14)$. Existem casos na literatura $(27 \%)$ de pacientes vivos e sem doença recidivada/a distância, aos $4,15,25$ e 48 meses de seguimento $(5,9,11)$, incluindo o caso relatado, que possui o maior intervalo livre de doença.

Dessa forma, o leiomiossarcoma primário da glândula tireoide, apesar de raro, constitui entidade diferente do carcinoma indiferenciado, deve estar presente no diagnóstico diferencial das neoplasias tireoideanas, é de tratamento eminentemente cirúrgico e possui um prognóstico sombrio, semelhante ao dos carcinomas indiferenciados, em razão das altas taxas de metástase a distância.

Declaração: os autores declaram não haver conflitos de interesse científico neste estudo.

\section{REFERÊNCIAS}

1. Liu CY, Wang MC, Li WY, Chang SY, Chu PY. Sarcoma of the larynx: treatment results and literature review. J Chin Med Assoc. 2006;69(3):120-4.

2. Perez Villa J, Muntané MJ, del Prado Venegas M, Viladot J. [Leiomyosarcoma of the larynx. Based on one case]. Acta Otorrinolaringol Esp. 2001.52(3):254-7.

3. Akcam T, Oysul K, Birkent H, Gerek M, Yetiser S. Leiomyosarcoma of the head and neck: report of two cases and review of the literature. Auris Nasus Larynx. 2005;32(2):209-12.

4. Just PA, Just PA, Guillevin R, Capron F, Le Charpentier M, Le Naour $\mathrm{G}$, et al. An unusual clinical presentation of a rare tumor of the thyroid gland: report on one case of leiomyosarcoma and review of literature. Ann Diagn Pathol. 2008;12(1):50-6.

5. Wang TS, Ocal IT, Oxley K, Sosa JA. Primary leiomyosarcoma of the thyroid gland. Thyroid. 2008;18(4):425-8.

6. Thompson LD, Wenig BM, Adair CF, Shmookler BM, Heffess CS. Primary smooth muscle tumors of the thyroid gland. Cancer. 1997;79(3):579-87.

7. Kawahara E, Nakanishi I, Terahata S, Ikegaki S. Leiomyosarcoma of the thyroid gland. A case report with a comparative study of five cases of anaplastic carcinoma. Cancer. 1988;62(12):2558-63.

8. lidaY, Katoh R, Yoshioka M, OyamaT, Kawaoi A. Primary leiomyosarcoma of the thyroid gland. Acta Pathol Jpn. 1993;43(1-2):71-5.

9. Ozaki O, Sugino K, MimuraT, Ito K, Tamai S, HosodaY. Primary leiomyosarcoma of the thyroid gland. Surg Today. 1997;27(2):177-80.

10. Tsugawa K, Koyanagi N, Nakanishi K, Wada H, Tanoue K, Hashizume $M$, et al. Leiomyosarcoma of the thyroid gland with rapid growth and tracheal obstruction: a partial thyroidectomy and tracheostomy using an ultrasonically activated scalpel can be safely performed with less bleeding. Eur J Med Res. 1999;4(11):483-7.

11. Chetty R, Clark SP, Dowling JP. Leiomyosarcoma of the thyroid: immunohistochemical and ultrastructural study. Pathology. 1993;25(2):203-5.

12. Day AS, Lou PJ, Lin WC, Chou CC. Over-expression of c-kit in a primary leiomyosarcoma of the thyroid gland. Eur Arch Otorhinolaryngol. 2007;264(6):705-8.

13. Takayama F, Matsuba H, Kobayashi S, Ito N, Sone S. MR imaging of primary leiomyosarcoma of the thyroid gland. Eur $\mathrm{J}$ Radiol. 2001;37(1):36-41.

14. Mansouri H, Gaye M, Errihani H, Kettani F, Gueddari BE. Leiomyosarcoma of the thyroid gland. Acta Otolaryngol. 2008;128(3):335-6.

15. Adachi M, Wellmann KF, Garcia R. Metastatic leiomyosarcoma in brain and heart. J Pathol. 1969;98(4):294-6.

16. Kaur A, Jayaram G. Thyroid tumors: cytomorphology of medullary, clinically anaplastic, and miscellaneous thyroid neoplasms. Diagn Cytopathol. 1990;6(6):383-9.

17. Ramakrishnan R, Pradhan SA, Desai S, Chinoy RF. Leiomyosarcoma masquerading as a thyroid mass in a 3-year-old child. Med Pediatr Oncol. 2002;38(2):131-2.

18. Tulbah A, Al-Dayel F, Fawaz I, Rosai J. Epstein-Barr virus-associated leiomyosarcoma of the thyroid in a child with congenital immunodeficiency: a case report. Am J Surg Pathol. 1999;23(4):473-6.

19. Thompson LD. Smooth muscle tumors. In: DeLellis RA, et al., editors. World Health Organization Classification of Tumours. Pathology and Genetics of Tumors of Endocrine Organs 2004, IARC Press: Lyon. p. 115. 